\title{
On Hermitian Forms over Dyadic Non-maximal Local Orders
}

\author{
Chia-Fu Yu
}

\begin{abstract}
We reduce a study of polarized abelian varieties over finite fields to the classification problem of skew-Hermitian modules over (possibly nonmaximal) local orders. The main result of this paper gives a complete classification of these skew-Hermitian modules for the case where the ground ring is a dyadic non-maximal local order.
\end{abstract}

Keywords: hermitians forms, abelian varieties, local orders.

\section{INTRODUCTION}

Let $p$ be a rational prime number. Let $R:=\mathbb{Z}_{2}[X] /\left(X^{2}+p\right)=\mathbb{Z}_{2}[\pi]$, an order of the $\mathbb{Q}_{2}$-algebra $E:=\mathbb{Q}_{2}[X] /\left(X^{2}+p\right)$, where $\mathbb{Z}_{2}$ is the ring of 2-adic integers, and $\pi$ is the image of $X$ in $R$. Denote by $a \mapsto \bar{a}$ the non-trivial involution on $E$ and $O_{E}$ the ring of integers in $E$. By a skew-Hermitian module over $R$ we mean a $\mathbb{Z}_{2}$-free finite $R$-module $M$ together with a $\mathbb{Z}_{2}$-valued non-degenerate alternating pairing

$$
\psi: M \times M \rightarrow \mathbb{Z}_{2}
$$

such that $\psi(a x, y)=\psi(x, \bar{a} y)$ for all $a \in R$ and $x, y \in M$. If $M$ is self-dual with respect to the pairing $\psi$, then it is called a self-dual skew-Hermitian $R$-module. In this paper we study the classification of self-dual skew-Hermitian modules over $R$. As an elementary fact, the ring $R$ is the maximal order if and only if $p=2$ or $p \equiv 1(\bmod 4)$. It is easier to handle the case where $R$ is maximal; the classification is known even for any non-Archimedean local maximal order of characteristic not equal to 2 , due to Jacobowitz [6]. We give an exposition of the 
classification in Section 2, for the reader's convenience. The main part of this paper treats the case where $R$ is not maximal, that is, the case $p \equiv 3(\bmod 4)$. We now describe the main results.

Let $r \geq 1$ be an integer. Let $S_{r}$ be the set of symmetric matrices in $\mathrm{GL}_{r}\left(\mathbb{F}_{2}\right)$. Define the equivalence relation $\sim$ on $S_{r}$ by $A \sim B$, for $A, B \in S_{r}$, if there exists a matrix $P \in \mathrm{GL}_{r}\left(\mathbb{F}_{2}\right)$ such that $B=P^{t} A P$. Denote by $S_{r} / \sim$ the set of equivalence classes in $S_{r}$. Define integers $m_{r}$ for $r \geq 0$ by $m_{0}:=1$ and

$$
m_{r}:=\# S_{r} / \sim, \quad \forall r \geq 1 .
$$

Theorem 1.1. Assume $p \equiv 7(\bmod 8)$. There are

$$
\sum_{r=0}^{n} m_{r}
$$

non-isomorphic self-dual skew-Hermitian $R$-modules of $\mathbb{Z}_{2}$-rank $2 n$.

Theorem 1.2. Assume $p \equiv 3(\bmod 8)$. There are

$$
\sum_{r=0}^{n} m_{r}
$$

non-isomorphic self-dual skew-Hermitian $R$-modules of $\mathbb{Z}_{2}$-rank $2 n$.

The proofs are given in Sections 4 and 5. Though the statements of Theorems 1.1 and 1.2 look the same, the structures in the classification are different. It is easy to compute the integers $m_{r}$; see Lemma 4.7. The classification problem for $\left(\mathbb{Z}_{2}\right.$-valued) skew-Hermitian $R$-modules is equivalent to the same problem for Hermitian $R$-modules. Indeed, let $(M, \psi)$ be a skew-Hermitian module over $R$. Then there is a unique Hermitian form

$$
\varphi: M \times M \rightarrow 2^{m} R
$$

such that

$$
\psi(x, y):=\operatorname{Tr}_{E / \mathbb{Q}_{2}} \pi \varphi(x, y), \quad \forall x, y \in M .
$$

Here $m$ is the largest integer such that $R^{\vee} \subset 2^{m} R$, where $R^{\vee}$ is the dual lattice of $R$ for the pairing $(a, b) \mapsto \operatorname{Tr}_{E / \mathbb{Q}_{2}}(a b)$; in fact $m=0$ or -1 depending on whether $R$ is maximal or not, cf. $\S 5.1$. Conversely, given a Hermitian $R$-module $(M, \varphi)$ we get a $\left(\mathbb{Z}_{2}\right.$-valued) skew-Hermitian $R$-module $(M, \psi)$ by setting

$$
\psi(x, y):=\operatorname{Tr}_{E / \mathbb{Q}_{2}} \pi \varphi(x, y), \quad \forall x, y \in M .
$$


It is worth noting that when $p \equiv 3(\bmod 4)$, the ground $\operatorname{ring} R$ is not hereditary (see Remark 3.4), nor the condition that $a+\bar{a}=1$ for some $a \in R$ is not fulfilled. Therefore, results in the paper complement those of Riehm [12], and a general Witt type cancellation theorem [1, Theorem 3] obtained by Bayer-Fluckiger and Fainsilber; see Propositions 4.4 and 5.7.

The motivation of this work is to determine the Tate modules of certain abelian varieties over finite fields as Galois modules. The reader who is not familiar with abelian varieties may consult the reference [9] by Mumford. An abelian variety $A$ over a field $k$ of characteristic $p$ is said to be superspecial if it is isomorphic to a product of supersingular elliptic curves over an algebraic closure $\bar{k}$ of $k$. Let $\Sigma_{n}\left(\mathbb{F}_{p}\right)$ denote the set of isomorphism classes of $n$-dimensional superspecial abelian varieties $(A, \lambda)$ over $\mathbb{F}_{p}$ together with a principal polarization $\lambda$ over $\mathbb{F}_{p}$ such that $\pi_{A}^{2}=-p$, where $\pi_{A}$ is the Frobenius endomorphism of $A$. Suppose $(A, \lambda)$ is an element in $\Sigma_{n}\left(\mathbb{F}_{p}\right)$. For any prime $\ell \neq p$, the associated Tate module $T_{\ell}(A)$ is a free $\mathbb{Z}_{\ell}$-module of rank $2 n$ together with a continuous action $\rho_{A}$ of the Galois group $\mathcal{G}:=\operatorname{Gal}\left(\overline{\mathbb{F}}_{p} / \mathbb{F}_{p}\right)$ and a Galois equivariant self-dual alternating pairing (the Weil pairing)

$$
e_{\ell}: T_{\ell}(A) \times T_{\ell}(A) \rightarrow \mathbb{Z}_{\ell}(1),
$$

where

$$
\mathbb{Z}_{\ell}(1):=\lim _{\leftarrow} \mu_{\ell^{m}}\left(\overline{\mathbb{F}}_{p}\right)
$$

is the Tate twist. Let $\sigma: x \mapsto x^{p}$ be the Frobenius automorphism of $\mathcal{G}$. Since $\sigma x=\pi_{A} x$ for all $x \in T_{\ell}(A)$, the action of the Galois group $\mathcal{G}$ on the Tate module $T_{\ell}(A)$ factors through the quotient

$$
\mathbb{Z}_{\ell}[\mathcal{G}] \rightarrow \mathbb{Z}_{\ell}[X] /\left(X^{2}+p\right)=\mathbb{Z}_{\ell}\left[\pi_{A}\right]
$$

The pairing $e_{\ell}$ induces an involution $a \mapsto \bar{a}$ on $\mathbb{Z}_{\ell}\left[\pi_{A}\right]$ by the adjoint. It follows from $\pi_{A} \bar{\pi}_{A}=p$ and $\pi_{A}^{2}=-p$ that this involution is non-trivial. Fix an isomorphism $\mathbb{Z}_{\ell}(1) \simeq \mathbb{Z}_{\ell}$ as $\mathbb{Z}_{\ell}$-modules. The problem of classifying Tate modules of superspecial abelian varieties in $\Sigma_{n}\left(\mathbb{F}_{p}\right)$ as $\mathcal{G}$-modules together to the Weil pairing amounts to

(1) classifying self-dual skew-Hermitian modules over the ring $\mathbb{Z}_{\ell}[X] /\left(X^{2}+p\right)$, and 
(2) determining the image of $\Sigma_{n}\left(\mathbb{F}_{p}\right)$ in the set of isomorphism classes of skew-Hermitian modules over $\mathbb{Z}_{\ell}[X] /\left(X^{2}+p\right)$.

The second problem seems to be hard. Besides, in order to state the result of the second problem, one needs an explicit description of the classification. In this paper we limit ourselves to the first problem.

As mentioned before, if the ring $\mathbb{Z}_{\ell}[X] /\left(X^{2}+p\right)$ is the maximal order in the

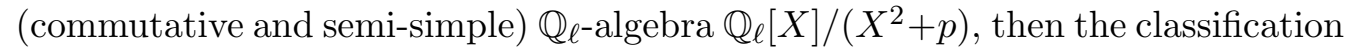
is known. As well-known the ring $\mathbb{Z}_{\ell}[X] /\left(X^{2}+p\right)$ is maximal except when $\ell=2$ and $p \equiv 3(\bmod 4)$, which is also the most complicated case. Theorems 1.1 and 1.2 solve the first problem in this exceptional case. An immediate consequence of Theorems 1.1 and 1.2 (also using Lemma 4.7) is the following result.

Theorem 1.3. Notation as above. Assume $p \equiv 3(\bmod 4)$. There are at most $n+\lfloor n / 2\rfloor$ isomorphism classes of polarized 2-adic Tate modules $\left(T_{2}(A), \rho_{A}, e_{2}\right)$ for all elements $(A, \lambda)$ in $\Sigma_{n}\left(\mathbb{F}_{p}\right)$.

The paper is organized as follows. In Section 2 we give an exposition of the classification of Hermitian forms over non-Archimedean local maximal orders of characteristic not equal to 2, following Jacobowitz [6]. Section 3 gives the complete classification of $R$-modules. Sections 4 and 5 treat the classification of skew-Hermitian $R$-modules in the split case $p \equiv 7(\bmod 8)$ and the inert case $p \equiv 3(\bmod 8)$ separately.

Acknowledgments. The manuscript is prepared during the author's stay at l'Institut des Hautes Études Scientifiques. He acknowledges the institution for kind hospitality and excellent working conditions. The research was partially supported by grants NSC 97-2115-M-001-015-MY3 and AS-99-CDA-M01. The author acknowledges the referee for his/her careful reading and helpful comments which remove many errors and inaccuracies.

\section{HeRmitian Forms OVER LOCAL FIELDS}

In this section we give an exposition of the classification of Hermitian forms over local maximal orders, for the reader's convenience. Our reference is Jacobowitz $[6]$. 
2.1. Hermitian spaces. Let $F$ be a non-Archimedean local field of char $F \neq 2$. Let $O_{F}$ be the ring of integers of $F$, and $\pi_{F}$ a uniformizer of $O_{F}$. Let $E$ be a quadratic field extension of $F$; $E$ is necessarily separable over $F$. Let $O_{E}$ be the ring of integers in $E$, and $\pi$ a uniformizer of $O_{E}$. Write $a \mapsto \bar{a}$ for the non-trivial automorphism of $E$ over $F$, and let $\pi=\pi_{F}$ if the extension $E / F$ is unramified. Let $v$ be the normalized valuation on $E$ such that $v(\pi)=1$.

By a Hermitian space over $E$ we mean a finite-dimensional vector $V$ over $E$, together with an $F$-bilinear pairing

$$
h: V \times V \rightarrow E
$$

such that $h(a x, b y)=a \bar{b} h(x, y)$ and $h(y, x)=\overline{h(x, y)}$ for all $a, b \in E$ and $x, y \in V$. The pairing $h$ is called a Hermitian form. It is called non-degenerate if the induced linear map $V \rightarrow V^{*}:=\operatorname{Hom}_{E}(V, E)$ by $x \mapsto h(\cdot, x)$ is injective (and hence isomorphic). By a Hermitian module over $O_{E}$ we mean a finite free $O_{E^{-}}$ module $L$ together with a Hermitian form $h$ on $V=E \otimes_{O_{E}} L$, not necessarily assumed that $h(L, L) \subset O_{E}$. It is called non-degenerate if the Hermitian $E$ space $(V, h)$ is non-degenerate; it is unimodular if $h(L, L) \subset O_{E}$ and the induced map $L \rightarrow L^{*}:=\operatorname{Hom}_{O_{E}}\left(L, O_{E}\right)$ is an isomorphism. A full rank $O_{E}$-submodule in a Hermitian $E$-space is usually called an $O_{E}$-lattice. A decomposition of a Hermitian module (resp. space) $L$ into submodules (resp. subspaces) $L_{1}$ and $L_{2}$ :

$$
L=L_{1} \oplus L_{2}
$$

means that $L=L_{1}+L_{2}, L_{1} \cap L_{2}=0$ and $h(x, y)=0$ for all $x \in L_{1}$ and $y \in L_{2}$. For a submodule (resp. subspace) $L_{1}$ of $L$, denote by $L_{1}^{\perp}$ the orthogonal complement of $L_{1}$ in $L$.

Lemma 2.1. Any Hermitian space $(V, h)$ over $E$ has a decomposition

$$
V=V^{\perp} \oplus V_{1},
$$

where $V_{1}$ is any E-linear subspace complementary to the null subspace $V^{\perp}$. The Hermitian subspace $V_{1}$, if non-zero, is non-degenerate and the projection $V_{1} \rightarrow$ $V / V^{\perp}$ is isometric.

From now on, we assume that $(V, h)$ is non-degenerate. The determinant or discriminant of $V$ (resp. of a lattice $L$ ), denoted by $d V$ (resp. $d L$ ), is defined as $\operatorname{det}\left(h\left(x_{i}, x_{j}\right)\right)$, where $x_{1}, \ldots, x_{n}$ is an $E$-basis for $V$ (resp. an $O_{E}$-basis for $L$ ). The determinant $d V$ (resp. $d L$ ) is unique up to an element in $N_{E / F}\left(E^{\times}\right.$) (resp. 
$N_{E / F}\left(O_{E}^{\times}\right)$). Write $d V_{1} \simeq d V_{2}$ (resp. $\left.d L_{1} \simeq d L_{2}\right)$ if there is an element $a \in$ $N_{E / F}\left(E^{\times}\right)\left(\right.$resp. $\left.a \in N_{E / F}\left(O_{E}^{\times}\right)\right)$such that $d V_{2}=a \cdot d V_{1}\left(\right.$ resp. $\left.d L_{2}=a \cdot d L_{1}\right)$.

\section{Theorem 2.2.}

(1) We have $V=E x_{1} \oplus \cdots \oplus E x_{n-1} \oplus E x_{n}$ with $h\left(x_{i}, x_{i}\right)=1$ for $i=$ $1, \ldots, n-1$ and $h\left(x_{n}, x_{n}\right)=d V$.

(2) Two Hermitian spaces $V_{1}$ and $V_{2}$ over $E$ are isometric if and only if $\operatorname{dim} V_{1}=\operatorname{dim} V_{2}$ and $d V_{1} \simeq d V_{2}$.

Proof. This is Theorem 3.1 of [6]. The statement (1) follows from the fact that any non-degenerate quadratic form of rank $\geq 4$ over $F$ represents every non-zero element in $F$. The statement (2) follows from (1).

2.2. General properties of Hermitian lattices. Let $(L, h)$ be a Hermitian lattice. For any elements $x_{1}, \ldots, x_{n}$ in $L$, denote by $<x_{1}, \ldots, x_{n}>_{O_{E}}$ the $O_{E^{-}}$ submodule generated by $x_{1}, \ldots, x_{n}$. If $L$ has an orthogonal basis $x_{1}, \ldots, x_{n}$ with $h\left(x_{i}, x_{i}\right)=\alpha_{i}$, we will write

$$
L=<x_{1}>\oplus \cdots \oplus<x_{n}>\simeq\left(\alpha_{1}\right) \oplus \cdots \oplus\left(\alpha_{n}\right) .
$$

\section{Definition 2.3.}

(1) A vector $x \in L$ is called maximal if $x \notin \pi L$.

(2) Let $s L:=\{h(x, y) \mid x, y \in L\}$, and let $n L$ be the $O_{E}$-submodule in $E$ generated by elements $h(x, x)$ for all $x \in L$. Clearly, one has $n L \subset s L$.

(3) We call $L$ normal if $n L=s L$, and subnormal otherwise.

(4) A lattice $L$ is called $\pi^{i}$-modular, where $i \in \mathbb{Z}$, if $h(x, L)=\left(\pi^{i}\right)$ for every maximal vector $x \in L ; L$ is called modular if it is $\pi^{i}$-modular for some $i \in \mathbb{Z}$.

Clearly, if $L_{1}$ and $L_{2}$ are both $\pi^{i}$-modular, then so is their direct sum $L_{1} \oplus L_{2}$. Any rank one lattice is modular. The lattice $L=<x, y>$ with $v(h(x, y))=$ $i, v\left(h(x, x)>i\right.$, and $v(h(y, y))>i$ is a $\pi^{i}$-modular plane. We write

$$
\left(\begin{array}{l}
a b \\
\bar{b} c
\end{array}\right)
$$


for the Hermitian plane $\left(O_{E}^{2}, h\right)$ with $h\left(e_{1}, e_{1}\right)=a, h\left(e_{1}, e_{2}\right)=b$ and $h\left(e_{2}, e_{2}\right)=c$. For any $i \in \mathbb{Z}$, define the hyperbolic plane $H(i)$ to be

$$
\left(\begin{array}{cc}
0 & \pi^{i} \\
\bar{\pi}^{i} & 0
\end{array}\right) .
$$

Proposition 2.4. There is an $O_{E}$-basis $x_{1}, \ldots, x_{r}, y_{1}, \ldots, y_{s}, z_{1}, \ldots, z_{s}$ such that

$$
V=<x_{1}>\oplus \cdots \oplus<x_{r}>\oplus<y_{1}, z_{1}>\oplus \cdots \oplus<y_{s}, z_{s}>
$$

and all components are modular.

Proof. This is Proposition 4.3 of [6].

Proposition 2.5. A $\pi^{i}$-modular lattice $L$ has an orthogonal basis if any of the following conditions holds:

(1) L has odd rank.

(2) $L$ is normal.

(3) $i=0$ and there is an element $a \in E$ such that $v(a)=v(a+\bar{a})=0$.

Proof. This is Proposition 4.4 of [6].

\section{Definition 2.6.}

(1) For any integer $j \in \mathbb{Z}$, define

$$
L_{(j)}:=\left\{x \in L \mid h(x, L) \subset\left(\pi^{j}\right)\right\} .
$$

This defines a decreasing filtration $\left\{L_{(j)}\right\}$ on $L$.

(2) A decomposition $L=\oplus_{1 \leq \lambda \leq t} L_{\lambda}$ of $L$ is called a Jordan splitting if each $L_{\lambda}$ is modular and

$$
s L_{1} \supsetneq \ldots, \supsetneq s L_{t} .
$$

Two Jordan splittings

$$
L=\oplus_{1 \leq \lambda \leq t} L_{\lambda}, \quad K=\oplus_{1 \leq \lambda \leq T} K_{\lambda}
$$

are said to be of the same type if $t=T$, and for each $\lambda$, one has

$$
s L_{\lambda}=s K_{\lambda}, \quad \operatorname{rank} L_{\lambda}=\operatorname{rank} K_{\lambda},
$$

and either both $L_{\lambda}$ and $K_{\lambda}$ are normal or both are subnormal. 
It follows from Proposition 2.4 that every lattice has a Jordan splitting. Any two Jordan splittings of a lattice $L$ are of the same type (see [6, p. 449]). For a Jordan splitting $L=\oplus_{\lambda} L_{\lambda}$, define the integers $s(\lambda)$ and $u(\lambda)=u_{L}(\lambda)$ for $\lambda=1, \ldots, t$ by

$$
s L_{\lambda}=\left(\pi^{s(\lambda)}\right), \quad n L_{(s(\lambda))}=\left(\pi^{u(\lambda)}\right),
$$

and the fractional $O_{E}$-ideals $\mathfrak{f}(\lambda)$ for $\lambda=1, \ldots, t-1$ by

$$
\mathfrak{f}(\lambda):=\left(\pi^{u(\lambda)+u(\lambda+1)-2 s(\lambda)}\right) .
$$

Since any two splittings are of the same type, the invariant $\{s(\lambda)\}$, and hence the invariants $\{u(\lambda)\}$ and $\{\mathfrak{f}(\lambda)\}$ are independent of the choice of Jordan splittings.

Theorem 2.7. Suppose $E / F$ is unramified. Then

(1) There is an element $a$ in $E$ such that $v(a)=v(a+\bar{a})=0$.

(2) Any $\pi^{i}$-modular lattice $L$ is isomorphic to $\left(\pi^{i}\right) \oplus \ldots, \oplus\left(\pi^{i}\right)$.

(3) Any two lattices are isometric if and only if they are of the same type.

Proof. This is Theorem 7.1 of [6].

Theorem 2.8. Suppose that $E / F$ is ramified and non-dyadic.

(1) Let $L$ be a $\pi^{i}$-modular lattice of rank $n$. If $i=2 d$ is even, then

$$
L \simeq\left(\pi^{d}\right) \oplus \cdots \oplus\left(\pi^{d}\right) \oplus\left(\pi^{-(n-1) d} d L\right) .
$$

If $i$ is odd, then $n$ is even and

$$
L \simeq H(i) \oplus \cdots \oplus H(i) .
$$

(2) Let $L$ and $K$ be two lattices with Jordan splittings $\oplus L_{\lambda}$ and $\oplus K_{\lambda}$, respectively. Then $L \simeq K$ if and only if $L$ and $K$ are of the same type and $d L_{j} \simeq d K_{j}$ for every index $j$ for which $s(j)$ is even.

Proof. These are Proposition 8.1 and Theorem 8.2 of [6].

Theorems 2.7 and 2.8 complete the classification in the cases where $E / F$ is unramified, or $E / F$ is ramified and non-dyadic. It remains to describe the ramified dyadic case. 
2.3. The ramified dyadic case: modular lattices. In the remaining of this section we assume that $E / F$ is ramified and dyadic.

Proposition 2.9. Suppose that $L$ is a $\pi^{i}$-modular lattice of rank $\geq 3$. Then

$$
L \simeq L_{0} \oplus H(i) \oplus \cdots \oplus H(i) .
$$

where $L_{0}$ is a $\pi^{i}$-modular lattice of rank one or two satisfying $n L_{0}=n L$.

Proof. This is Proposition 10.3 of [6].

Proposition 2.10. Let $L_{1}$ and $L_{2}$ be two $\pi^{i}$-modular lattices. If $L_{1} \oplus H(i) \simeq$ $L_{2} \oplus H(i)$, then $L_{1} \simeq L_{2}$.

Proof. This is Proposition 9.3 of [6].

By Propositions 2.9 and 2.10, the classification of modular lattices is reduced to the case of planes. Furthermore we may assume that $i=0$ or 1 .

We need a classification of dyadic ramified extensions. For an element $a$ in $O O_{F}^{\times}$, denote by $\mathfrak{d}_{F}(a)$ the smallest $O_{F}$-ideal $J$ such that $a \bmod J$ is a square. If $a$ is a square, then define $\mathfrak{d}_{F}(a)$ to be (0). Since the squaring $x \mapsto x^{2}$ is an automorphism on $O_{F} /\left(\pi_{F}\right)$, one has $\mathfrak{d}_{F}(a) \subset\left(\pi_{F}\right)$ for any $a \in O_{F}^{\times}$. It is known that the $O_{F}$-ideals occurring as $\mathfrak{d}_{F}(a)$ for some $a \in O_{F}^{\times}$are precisely (0), (4), and all $\left(\pi_{F}^{2 k+1}\right)$ with $0<2 k+1<v_{F}(4)$ where $v_{F}\left(\pi_{F}\right)=1$. Furthermore, one has

$$
\mathfrak{d}_{F}\left(1+\pi_{F}^{2 k+1} \delta\right)=\left(\pi_{F}^{2 k+1}\right)
$$

for every $\delta \in O_{F}^{\times}$and every integer $k$ such that $0<2 k+1<v_{F}(4)$.

Write $E=F(\sqrt{\theta})$, where $\theta$ is a non-square unit or a prime element. We have the following two cases [6, p. 451]:

(a) $\theta$ is a prime element, or

(b) $\theta$ is a unit and $\mathfrak{d}_{F}(\theta)=\left(\pi_{F}^{2 k+1}\right)$ with $0<2 k+1<v_{F}(4)$. In this case,

$$
E=F\left(\left(1+\pi_{F}^{2 k+1} \delta\right)^{1 / 2}\right)
$$

for some unit $\delta \in O_{F}^{\times}$.

Note that the case $\mathfrak{d}_{F}(\theta)=(4)$ occurs only when $E / F$ is unramified. We refer the case (a) as ramified prime, ("R-P"), and the case (b) as ramified unit, ("R-U"). 
We use a notation from [6, p. 450]: to indicate an unspecified element $a$ in $E$ with $v(a) \geq v(b)$, we shall write $a=\{b\}$; to indicate an unspecified element $a$ with $v(a)=v(b)$, write $a=[b]$.

\section{Proposition 2.11.}

(1) If $L$ is $\pi^{i}$-modular, then $n L \supset n H(i)$.

(2) If $a \in F$ is any element in $n H(i)$, then the $\pi^{i}$-modular lattice $\left(\begin{array}{cc}0 & \pi^{i} \\ \bar{\pi}^{i} & a\end{array}\right)$ is isomorphic to $H(i)$.

Proof. This is Proposition 9.1 of [6].

Proposition 2.12. Let $L$ be $a \pi^{i}$-modular plane, where $i=0$ or 1 , with $n L=$ $n H(i)$.

(1) If $L$ is isotropic, then $L \simeq H(i)$.

(2) Either in $R-P$ with $i=1$ or in $R$ - $U$ with $i=0$, the lattice $L$ must be isotropic; particularly one has $L \simeq H(i)$. In the other two cases, if $L$ is anisotropic, then $(h(x, x))=n L$ for any maximal vector $x \in L$.

(3) If $K$ is another $\pi^{i}$-modular plane, with $n K=n L$ and $d K \simeq d L$, then one has $K \simeq L$.

Proof. This is Proposition 9.2 of [6].

Propositions 2.11 and 2.12 handle the case where $L$ is a $\pi^{i}$-modular plane with $n L=n H(i)$, the minimal case. The other case $n L \neq n H(i)$ is treated in the following proposition.

Proposition 2.13. Let $L$ be $a \pi^{i}$-modular plane, where $i=0$ or 1 , with $n L=$ $\left(\pi^{2 m}\right) \supsetneq n H(i)$.

(1) If $L$ is normal, then $L \simeq(1) \oplus(d L) \simeq\left(\begin{array}{cc}1 & 1 \\ 1 & \{1\}\end{array}\right)$.

(2) If $L$ is subnormal, then

$$
L \simeq\left(\begin{array}{cc}
\pi_{F}^{m} & \pi^{i} \\
\bar{\pi}^{i} & \{a\}
\end{array}\right)
$$

where $a=4 \pi_{F}^{-m+i}$ in $R-P$, and $a=4 \pi_{F}^{-2 k-m+i-1}$ in $R-U$. 
Proof. This is Proposition 10.2 of [6].

Using Propositions 2.9-2.13, one can obtain the following characterization for modular lattices (see [6, Proposition 10.4]).

Theorem 2.14. Let $L$ and $K$ be two $\pi^{i}$-modular lattices for some $i \in \mathbb{Z}$. Then $L \simeq K$ if and only if $\operatorname{rank} L=\operatorname{rank} K, n L=n K$, and $d L \simeq d K$.

2.4. The ramified dyadic case: the invariants. Let $L$ and $K$ be two Hermitian lattices, and let $I \subset O_{E}$ be a proper ideal. We write $d L / d K \simeq 1(\bmod I)$

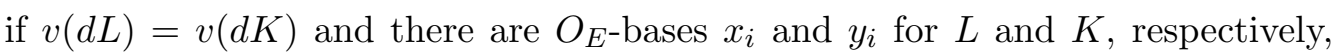
such that

$$
\operatorname{det}\left(h_{L}\left(x_{i}, x_{j}\right)\right) / \operatorname{det}\left(h_{K}\left(y_{i}, y_{j}\right)\right) \equiv 1 \quad(\bmod I) .
$$

The following theorem [6, Theorem 11.4] gives a complete classification of Hermitian forms over local maximal orders by the invariants.

Theorem 2.15. Let $L$ and $K$ be two Hermitian lattices. Suppose

$$
L=\oplus_{1 \leq \lambda \leq t} L_{\lambda} \quad \text { and } \quad K=\oplus_{1 \leq \lambda \leq T} K_{\lambda}
$$

are any Jordan splittings of $L$ and $K$, respectively. Then $L$ and $K$ are isometric if and only if the following four necessary conditions hold:

(1) $L$ and $K$ are of the same type.

(2) $d L \simeq d K$.

(3) $u_{L}(\lambda)=u_{K}(\lambda)$ for all $\lambda=1, \ldots, t$.

(4) For all $j=1, \ldots, t-1$, one has

$$
d\left(L_{1} \oplus \cdots \oplus L_{j}\right) / d\left(K_{1} \oplus \cdots \oplus K_{j}\right) \simeq 1 \quad(\bmod \mathfrak{f}(j)) .
$$

\section{UNPOLARIZED CASES}

In this section we assume that $p \equiv 3(\bmod 4)$. Recall that $R=\mathbb{Z}_{2}[X] /\left(X^{2}+\right.$ $p)=\mathbb{Z}_{2}[\pi]$ and $E=R \otimes_{\mathbb{Z}_{2}} \mathbb{Q}_{2}=\mathbb{Q}_{2}[\pi]$. Let $O_{E}$ be the ring of integers of $E$; $O_{E}$ is either a complete discrete valuation ring or is isomorphic to $\mathbb{Z}_{2} \times \mathbb{Z}_{2}$. Put $\alpha:=(\pi-1) / 2$. Then $\alpha \in O_{E}$ and

$$
O_{E}=\mathbb{Z}_{2}[\alpha]=\mathbb{Z}_{2}[X] /\left(X^{2}+X+(p+1) / 4\right) .
$$


Put $\omega:=\pi-1$, and one has

$$
R=\mathbb{Z}_{2}[\omega]=\mathbb{Z}_{2}[X] /\left(X^{2}+2 X+(p+1)\right) \quad \text { and } 2 \alpha=\omega .
$$

We shall classify $R$-modules $M$ which are free $\mathbb{Z}_{2}$-modules of finite rank. Write $<x_{1}, \ldots, x_{m}>_{R}$ for the $R$-submodule of $M$ generated by elements $x_{1}, \ldots, x_{m}$.

We divide the classification into two cases:

Case $(\mathbf{a}): p \equiv 3(\bmod 8)$. In this case, the algebra $E$ is an unramified quadratic extension of $\mathbb{Q}_{2}$. We have (at least) two indecomposable $\mathbb{Z}_{2}$-free finite $R$-modules: $R$ and $O_{E}$ as $R$-modules. The $R$-module structure of $O_{E}$ is given as follows: write $O_{E}=<1, \alpha>_{\mathbb{Z}_{2}}$, then

$$
\omega 1=2 \alpha \quad \text { and } \quad \omega \alpha=-2 \alpha-(p+1) / 2 .
$$

If $M$ is an $R$-module of the form $R^{\oplus r} \oplus O_{E}^{\oplus s}$ for some non-negative integers $r$ and $s$, then the integers $r$ and $s$ are uniquely determined by $M$. Indeed, we have $r+s=\operatorname{dim}_{E} M \otimes_{\mathbb{Z}_{2}} \mathbb{Q}_{2}$, and $M /(2, \omega) M=\left(\mathbb{F}_{2}\right)^{r} \oplus\left(\mathbb{F}_{2} \oplus \mathbb{F}_{2}\right)^{s}$.

Case (b): $p \equiv 7(\bmod 8)$. In this case, the algebra $E$ is isomorphic to $\mathbb{Q}_{2} \times \mathbb{Q}_{2}$. Let $\alpha_{1}$ and $\alpha_{2}$ are the roots of the quadratic polynomial $X^{2}+X+(p+1) / 4$ in $\mathbb{Z}_{2}$. By switching the order, we may assume that $\alpha_{1}$ is a unit and $\alpha_{2} \in 2 \mathbb{Z}_{2}$. By the Chinese Remainder Theorem, we have the isomorphisms

$$
O_{E}=\mathbb{Z}_{2}[\alpha] \simeq O_{E} /\left(\alpha-\alpha_{1}\right) \times O_{E} /\left(\alpha-\alpha_{2}\right) \simeq \mathbb{Z}_{2} \times \mathbb{Z}_{2} .
$$

Clearly, $X^{2}+2 X+(p+1)=\left(X-2 \alpha_{1}\right)\left(X-2 \alpha_{2}\right)$ and $\left(\omega-2 \alpha_{1}\right)\left(\omega-2 \alpha_{2}\right)=0$ in $R$. There are (at least) three indecomposable $\mathbb{Z}_{2}$-free finite $R$-modules:

$$
R, \quad R /\left(\omega-2 \alpha_{1}\right), \quad \text { and } R /\left(\omega-2 \alpha_{2}\right) .
$$

Among them, one has

$$
O_{E} \simeq R /\left(\omega-2 \alpha_{1}\right) \oplus R /\left(\omega-2 \alpha_{2}\right)
$$

as $R$-modules. If $M$ is an $R$-module of the form $R^{r} \oplus\left[R /\left(\omega-2 \alpha_{1}\right)\right]^{s} \oplus\left[R /\left(\omega-2 \alpha_{2}\right)\right]^{t}$ for some non-negative integers $r, s$ and $t$, then the integers $r, s$ and $t$ are uniquely determined by $M$. Indeed, we have

$$
\operatorname{rank}_{\mathbb{Z}_{2}} M=2 r+s+t, \quad M /(2, \omega) M=\mathbb{F}_{2}^{r} \oplus \mathbb{F}_{2}^{s} \oplus \mathbb{F}_{2}^{t},
$$


and

$$
M /\left(\omega-2 \alpha_{1}\right) M=\left[R /\left(\omega-2 \alpha_{1}\right)\right]^{r+s} \oplus\left(\mathbb{F}_{2}\right)^{t} .
$$

Conversely, we shall show that the indecomposable finite $R$-modules described in Cases (a) and (b) exhaust all possibilities.

Theorem 3.1. Let $M$ be a $\mathbb{Z}_{2}$-free finite $R$-module. Then

(1) Assume $p \equiv 3(\bmod 8)$ (Case $(\mathrm{a}))$. The $R$-module $M$ is isomorphic to $R^{r} \oplus O_{E}^{s}$ for some non-negative integers $r$ and $s$. Moreover, the integers $r$ and $s$ are uniquely determined by $M$.

(2) Assume $p \equiv 7(\bmod 8)$ (Case $(\mathrm{b}))$. The $R$-module $M$ is isomorphic to

$$
R^{r} \oplus\left[R /\left(\omega-2 \alpha_{1}\right)\right]^{s} \oplus\left[R /\left(\omega-2 \alpha_{2}\right)\right]^{t}
$$

for some non-negative integers $r, s$ and $t$. Moreover, the integers $r, s$ and $t$ are uniquely determined by $M$.

Proof. We have seen that the integers $r, s$ (and $t$ for (2)) are uniquely determined by the $R$-module $M$. We now prove the first part of each statement.

(1) Assume $p \equiv 3(\bmod 8)$. In this case we have $M \otimes_{\mathbb{Z}_{2}} \mathbb{Q}_{2}=M \otimes_{R} E \simeq E^{n} \simeq$ $\mathbb{Q}_{2}^{2 n}$, and hence $M \simeq \mathbb{Z}_{2}^{2 n}$ and $\bar{M}:=M / 2 M \simeq \mathbb{F}_{2}^{2 n}$. Let

$$
\bar{M}:=M / 2 M=\left(\mathbb{F}_{2}[\omega] / \omega^{2}\right)^{r} \oplus\left(\mathbb{F}_{2}\right)^{2 s}
$$

be the decomposition as $R / 2 R=\mathbb{F}_{2}[\omega] /\left(\omega^{2}\right)$-modules. We first show that if $s=0$, then $M \simeq R^{r}$. Since $r=\operatorname{dim} M \otimes_{R} \mathbb{F}_{2}=\operatorname{dim}_{E} M \otimes_{R} E$ and $R$ is a local Noetherian domain, the module $M$ is free.

Now suppose $s>0$. Choose an element $a \neq 0 \in\left(\mathbb{F}_{2}\right)^{2 s}$ and let $x \in M$ be an element such that $\bar{x}=a$. As $\overline{\omega x}=0$, the element $\omega x / 2 \in M$. Put $M_{1}:=<x, \omega x / 2>_{\mathbb{Z}_{2}}$; it is an $R$-module and is isomorphic to $O_{E}$. Let $\omega^{\prime}$ denote the conjugate of $\omega$; one has $\omega^{\prime}=-2-\omega$ and $\omega \omega^{\prime}=(1+p)$. Note that $(1+p) / 4$ is a unit. Since $\bar{x} \notin \omega^{\prime} \bar{M}$, one has $x \notin \omega^{\prime} M$. We show that $\overline{\omega x / 2} \neq 0$. Suppose not, then $\omega x=4 y$ for some $y \in M$. Applying $\omega^{\prime}$, we get $x=\omega^{\prime} y^{\prime}$ for some $y^{\prime} \in M$, contradiction. We show that the $\mathbb{F}_{2}$-vector space $\bar{M}_{1}:=M_{1} / 2 M=<$ $\bar{x}, \overline{\omega x / 2}>$ is 2-dimensional. Suppose that $x+\omega x / 2=2 z$ for some $z \in M$. Since $(1+\omega / 2)=-\omega^{\prime} / 2$, we get $x=-\left[4 /\left(\omega \omega^{\prime}\right)\right] \cdot \omega z \in \omega M$ and $\bar{x} \in \omega \bar{M}=\omega^{\prime} \bar{M}$, contradiction. Therefore, the quotient $\bar{M} / \bar{M}_{1}$ has dimension decreased by 2 . On 
the other hand, the $\mathbb{Z}_{2}$-rank of $M / M_{1}$ also decreases by 2 . This shows that $M / M_{1}$ is free as $\mathbb{Z}_{2}$-modules. If the integer $s$ in (3.2) for $M / M_{1}$ is positive, then we can find an $R$-submodule $M_{2}=<x_{2}, \omega x_{2} / 2>_{\mathbb{Z}_{2}} \simeq O_{E}$ not contained in the vector space $E \otimes_{O_{E}} M_{1}$ such that $M /\left(M_{1}+M_{2}\right)$ is free as $\mathbb{Z}_{2}$-modules. Continuing this process, we get $R$-submodules $M_{1}, \ldots, M_{s^{\prime}}$, which are isomorphic to $O_{E}$, such that $M_{1}+\cdots+M_{s^{\prime}}=M_{1} \oplus \cdots \oplus M_{s^{\prime}}$ and $M /\left(M_{1}+\cdots+M_{s^{\prime}}\right)$ is a free $R$-module. It follows that $M \simeq O_{E}^{s^{\prime}} \oplus R^{r^{\prime}}$. Since $s^{\prime}$ and $r^{\prime}$ are uniquely determined by $M$ as before, the integers $s^{\prime}$ and $r^{\prime}$ are actually equal to $s$ and $r$ in (3.2), respectively. This proves (1).

(2) Assume $p \equiv 7(\bmod 8)$. Let

$$
M_{1}:=\left\{x \in M \mid\left(\omega-2 \alpha_{1}\right) x=0\right\},
$$

and

$$
M_{2}:=\left\{x \in M \mid\left(\omega-2 \alpha_{2}\right) x=0\right\}
$$

Using the relation

$$
2=\left(\omega-2 \alpha_{1}\right)\left(2 \alpha_{2}+1\right)^{-1}-\left(\omega-2 \alpha_{2}\right)\left(2 \alpha_{2}+1\right)^{-1},
$$

one shows that $2 M \subset M_{1}+M_{2}$, and hence the quotient $M /\left(M_{1}+M_{2}\right)$ is an $\mathbb{F}_{2^{-}}$ vector space, say of dimension $r$. Let $x_{1}, \ldots, x_{r}$ be elements of $M$ such that the images $\bar{x}_{1}, \ldots, \bar{x}_{r}$ form an $\mathbb{F}_{2}$-basis for $M /\left(M_{1}+M_{2}\right)$. Put $F_{0}:=<x_{1}, \ldots, x_{r}>_{R}$, which is isomorphic to $R^{r}$, as $\bar{x}_{i}^{\prime} s$ form a basis for $F_{0} /\left(M_{1}+M_{2}\right)=F_{0} /(2, \omega) F_{0} \simeq$ $\mathbb{F}_{2}^{r}$. Now $\left(\omega-2 \alpha_{2}\right) F_{0} \subset M_{1}$, we choose elements $y_{1}, \ldots, y_{s}$ in $M_{1}$ so that the images $\bar{y}_{1}, \ldots, \bar{y}_{s}$ form an $R /\left(\omega-2 \alpha_{1}\right)$-basis for $M_{1} /\left(\omega-2 \alpha_{2}\right) F_{0}$, and put $F_{1}=<$ $y_{1}, \ldots, y_{s}>_{R}$. We have

$$
M_{1}=\left(\omega-2 \alpha_{2}\right) F_{0} \oplus F_{1}, \quad \text { and } \quad F_{0} \cap F_{1}=0 .
$$

Similarly, we have a free $R /\left(\omega-2 \alpha_{2}\right)$-submodule $F_{2}$ of $M_{2}$, of rank $t$, such that

$$
M_{2}=\left(\omega-2 \alpha_{1}\right) F_{0} \oplus F_{2}, \quad \text { and } \quad F_{0} \cap F_{2}=0 .
$$

We have $\left(F_{0}+F_{1}\right) \cap F_{2}=F_{0} \cap F_{2}=0$ and $M=F_{0}+F_{1}+F_{2}$, and hence $M=F_{0} \oplus F_{1} \oplus F_{2}$. This proves (2).

Corollary 3.2. Assume $p \equiv 3(\bmod 4)$. Let $A$ be an $n$-dimensional superspecial abelian variety $A$ over $\mathbb{F}_{p}$ with $\pi_{A}^{2}=-p$. Then the Tate module $T_{2}(A)$ of $A$ is isomorphic to $R^{r} \oplus O_{E}^{s}$ for some non-negative integers $r$ and s such that $r+s=n$. Moreover, the integers $r$ and $s$ are uniquely determined by $T_{2}(A)$. 
Proof. Note that the Tate space $V_{2}(A)=T_{2}(A) \otimes_{\mathbb{Z}_{2}} \mathbb{Q}_{2}$ is a free $E$-module of rank $n$; this follows from the fact that $\operatorname{tr}\left(a ; V_{2}(A)\right)=n \operatorname{tr}(a ; E)$ for all $a \in R$. It follows that the numbers $s$ and $t$ in Theorem 3.1 (2) above are the same. Therefore, the corollary follows.

Lemma 3.3. Assume $p \equiv 3(\bmod 4)$. Let $n \geq 1$ be an integer. For any nonnegative integers $r$ and $s$ with $r+s=n$, there exists an $n$-dimensional superspecial abelian variety $A_{r}$ over $\mathbb{F}_{p}$ with $\pi_{A}^{2}=-p$ such that the Tate module $T_{2}\left(A_{r}\right)$ of $A_{r}$ is isomorphic to $R^{r} \oplus O_{E}^{s}$.

Proof. Choose a supersingular elliptic curve $E_{0}$ over $\mathbb{F}_{p}$ such that the endomorphism ring $\operatorname{End}_{\mathbb{F}_{p}}\left(E_{0}\right)$ is equal to the ring $O_{\mathbb{Q}(\sqrt{-p})}$ of integers in the imaginary quadratic field $\mathbb{Q}(\sqrt{-p})$, and a supersingular elliptic curve $E_{1}$ over $\mathbb{F}_{p}$ such that the endomorphism ring $\operatorname{End}_{\mathbb{F}_{p}}\left(E_{0}\right)$ is equal to $\mathbb{Z}[\sqrt{-p}]$ (see Waterhouse [17, Theorem $4.2(3)$, p. 539]). Put $A_{r}=E_{1}^{r} \times E_{0}^{s}$, then the superspecial abelian variety $A_{r}$ has the desired property.

Remark 3.4. We recall that a (not necessarily commutative) ring $\Lambda$ is said to be left hereditary if every left ideal of $\Lambda$ is a projective $\Lambda$-module [11, Section $2 \mathrm{f}$, p. 27]. One defines in a similar manner for the notion of right hereditary. If $\Lambda$ is both left and right Noetherian, then $\Lambda$ is left hereditary if and only if it is right hereditary [11, p. 29]. In this case $\Lambda$ is simply called hereditary. Suppose that $A$ is a Dedekind domain with quotient field $K$ so that $A \neq K$ and $B$ is a finitedimensional semi-simple $K$-algebra. A hereditary $A$-order in $B$ is an $A$-order $\Lambda$ which is hereditary; note that $\Lambda$ is both left and right Noetherian as it is finite as an $A$-module. Write $B=\prod_{i=1}^{r} B_{i}$ as the product of its simple factors and let $A_{i}$ be the integral closure of $A$ in the center $K_{i}$ of $B_{i}$. Under the assumption that each $A_{i}$ is a finite $A$-module, any hereditary $A$-order $\Lambda$ in $B$ has the form $\Lambda=\prod_{i=1}^{r} \Lambda_{i}$, where $\Lambda_{i}$ is a hereditary $A_{i}$-order in $B_{i}$; cf. [11, Theorems 40.7, p. 369]. The finiteness assumption is used to make sure that any $A_{i}$-order in $B_{i}$ is also an $A$-order. This assumption is fulfilled if $A$ is Japanese or $B$ is separable over $K$, that is, the center of $B$ is a finite product of separable field extensions of $K$. We refer the reader to [8] for the definition of Japanese rings. Note that Theorem 40.7 of [11] is proved under the stronger assumption that $B$ is separable over $K$; however, the proof only uses the finiteness assumption as stated. 
As a special case, if $B$ is separable and commutative over $K$, then the maximal $A$-order in $B$ (it exists under the finiteness assumption) is the unique hereditary $A$-order in $B$.

From the discussion above, the non-maximal order $R=\mathbb{Z}_{2}[\pi]$ is not hereditary.

\section{Proof of Theorem 1.1.}

4.1. In this section we assume that $p \equiv 7(\bmod 8)$. Thus, the algebra $E$ is isomorphic to $\mathbb{Q}_{2} \times \mathbb{Q}_{2}$. Write $\sigma_{i}: E \rightarrow \mathbb{Q}_{2}$ for the $i$ th projection for $i=1,2$. Let $(M, \psi)$ be a self-dual skew-Hermitian $R$-module of $\mathbb{Z}_{2}$-rank $2 n$ and let $V:=$ $M \otimes_{\mathbb{Z}_{2}} \mathbb{Q}_{2}$. For $i=1,2$, let

$$
V^{i}:=\left\{x \in V ; a x=\sigma_{i}(a) x, \forall a \in E\right\} \quad \text { and } \quad M^{i}:=V^{i} \cap M
$$

be the $\sigma_{i}$-components of $V$ and $M$, respectively. It follows from the property $\psi(a x, y)=\psi(x, \bar{a} y)$ that each $V^{i}$ is an isotropic subspace over $\mathbb{Q}_{2}$. Thus, $\operatorname{dim} V^{i} \leq$ $n$ for $i=1,2$ and hence $\operatorname{dim} V^{1}=\operatorname{dim} V^{2}$. This shows that $V$ is a free $E$-module of rank $n$. Therefore, by Theorem 3.1, there are unique non-negative integers $r$ and $s$ with $r+s=n$ such that

$$
M \simeq R^{\oplus r} \oplus O_{E}^{\oplus s}
$$

as $R$-modules. Note that $r=0$ if and only if $M=M^{1}+M^{2}$, and we always have $M /\left(M^{1}+M^{2}\right) \simeq(\mathbb{Z} / 2 \mathbb{Z})^{r}$.

Define a self-dual skew-Hermitian $R$-module $\left(L_{h}, \psi_{h}\right)$ as follows. The $R$-module $L_{h}$ is $O_{E}=\mathbb{Z}_{2} \oplus \mathbb{Z}_{2}$. Put $e_{1}=(1,0)$ and $e_{2}=(0,1)$, and set $\psi_{h}\left(e_{1}, e_{2}\right)=1$.

4.2. Suppose that there exist elements $x \in M^{1}$ and $y \in M^{2}$ such that $\psi(x, y)=1$. Then the submodule $M_{1}=\langle x, y\rangle_{R}$ generated by $x$ and $y$ is isomorphic to $L_{h}$ as skew-Hermitian $R$-modules and we have the decomposition

$$
M=M_{1} \oplus M_{1}^{\perp}
$$

as skew-Hermitian $R$-modules, where $M_{1}^{\perp}$ is the orthogonal complement of $M_{1}$. Therefore, if $s=n$, then $M \simeq L_{h}^{\oplus n}$ as skew-Hermitian $R$-modules. 
4.3. Recall that the polarization type of a non-degenerate alternating pairing $\psi^{\prime}$ on a free module $M^{\prime}$ over a PID $R^{\prime}$ of rank $r$ is a tuple $\left(d_{1}, \ldots, d_{r}\right)$ of elements in $R^{\prime}$ with $d_{1}|\ldots| d_{r}$ such that there exists a Lagrangian $R^{\prime}$-basis $x_{i}, y_{i}$ for $i=1, \ldots, r$ such that $\psi^{\prime}\left(x_{i}, y_{i}\right)=d_{i}$. The elements $d_{i}$ are unique up to a unit in $R^{\prime}$. The choice of the Lagrangian basis $\left\{x_{i}, y_{i}\right\}$ gives rise to a splitting of $M^{\prime}=M_{1}^{\prime} \oplus M_{2}^{\prime}$ into isotropic submodules $M_{1}^{\prime}:=<x_{1}, \ldots, x_{r}>_{R^{\prime}}$ and $M_{2}^{\prime}:=<y_{1}, \ldots, y_{r}>_{R^{\prime}}$. Conversely, if $M^{\prime}$ splits into the direct sum of two isotropic submodules $M_{1}^{\prime}$ and $M_{2}^{\prime}$. then a Lagrangian basis $\left\{x_{i}, y_{i}\right\}$ can be chosen so that $x_{i} \in M_{1}^{\prime}$ and $y_{i} \in M_{2}^{\prime}$ for all $i=1, \ldots, r$.

\section{Lemma 4.1.}

(1) The polarization type of the pairing $\psi$ on the $R$-submodule $M^{1}+M^{2}$ viewed as a $\mathbb{Z}_{2}$-module is $(1, \ldots, 1,2, \ldots, 2)$, with multiplicity $s$ and $r$ for 1 and 2 respectively.

(2) There is a decomposition as skew-Hermitian R-modules

$$
M \simeq M_{1} \oplus L_{h}^{\oplus s},
$$

where $M_{1}$ is a free $R$-submodule of rank $r$ which is self-dual with respect to the pairing $\psi$.

Proof. The polarization type of the pairing $\psi$ on the submodule $M^{1}+M^{2}$ viewed as a $\mathbb{Z}_{2}$-module has the form $\left(2^{a_{1}}, \ldots, 2^{a_{n}}\right)$ with integers $0 \leq a_{1} \leq \cdots \leq$ $a_{n}$. Since $\left|M /\left(M^{1}+M^{2}\right)\right|=2^{r}$, one has $\sum_{i=1}^{n} a_{i}=r$. If $a_{1}>0$, then $a_{i}=1$ for all $i$ and $r=n$. Below we show that $s=0$ implies $a_{1}>0$. This proves the case where $s=0$.

If $a_{1}=0$, then there exist elements $x \in M^{1}$ and $y \in M^{2}$ such that $\psi(x, y)=1$. Using $\S 4.2$, one has a decomposition of $M$ into skew-Hermitian $R$-modules

$$
M \simeq M^{\prime} \oplus L_{h}
$$

with $M^{\prime} \simeq R^{r} \oplus O_{E}^{s-1}$ as an $R$-module, particularly $s>0$.

Suppose $s>0$ and we prove the statement by induction on $s$. Then $a_{1}=0$ and by the same argument we have $M \simeq M^{\prime} \oplus L_{h}$ as above. By the induction hypothesis, the polarization type of $\psi$ on $M^{1}+M^{2}$ is $(1, \ldots, 1,2, \ldots, 2)$ with multiplicity $s$ for 1 , and we get a decomposition of $M$ into skew-Hermitian $R$ modules

$$
M \simeq M_{1} \oplus L_{h}^{\oplus s},
$$


where $M_{1}$ is a free $R$-submodule of rank $r$ which is self-dual with respect to the pairing $\psi$. This proves both (1) and (2).

4.4. Now we classify self-dual skew-Hermitian $R$-modules $(M, \psi)$ in the case where $M \simeq R^{r}$, where $r$ is a positive integer. Let $N$ be the smallest $O_{E}$-module in $V$ containing $M$, and let $N^{\prime}=M^{1}+M^{2}$. We have

$$
N=N^{1} \oplus N^{2}, \quad N^{\prime}=2 N .
$$

The polarization type of $\psi$ on $2 N$ is $(2, \ldots, 2)$. Put $\psi_{N}:=2 \psi$. Then we have $\psi_{N}(N, N) \subset \mathbb{Z}_{2}$ and $N$ is self-dual with respect to the pairing $\psi_{N}$. Put $\bar{N}=$ $N / 2 N$ and let $\bar{\psi}_{N}: \bar{N} \times \bar{N} \rightarrow \mathbb{F}_{2}$ be the induced non-degenerate pairing. We have

$$
2 N \subset M \subset N, \quad \operatorname{dim}_{\mathbb{F}_{2}} \bar{M}=r
$$

and that $\bar{M}$ is isotropic for $\bar{\psi}_{N}$, where $\bar{M}:=M / 2 N$. Note that $M$ generates $N$ over $O_{E}$, or equivalently, $\bar{M}$ generates $\bar{N}$ over $O_{E} / 2 O_{E}=\mathbb{F}_{2} \times \mathbb{F}_{2}$.

Suppose $M_{1}$ is another self-dual skew-Hermitian $R$-module such that $M_{1} \simeq$ $R^{r}$. Define $N_{1}$ and $\psi_{N_{1}}$ similarly. Since $\left(N_{1}, \psi_{N_{1}}\right)$ is isomorphic to $\left(N, \psi_{N}\right)$ by Lemma 4.1 (2), we choose an isomorphism $\alpha:\left(N_{1}, \psi_{N_{1}}\right) \simeq\left(N, \psi_{N}\right)$ of skewHermitian $R$-modules. The image $M^{\prime}=\alpha\left(M_{1}\right)$ is an $R$-module which satisfies the same property (4.6) as $M$ does.

Now we fix the self-dual skew-Hermitian $R$-module $\left(N, \psi_{N}\right)$. Let $X_{r}$ the set of all $R$-submodules $M$ of $N$ such that

- $2 N \subset M \subset N$ and $\operatorname{dim}_{\mathbb{F}_{2}} \bar{M}=r$, where $\bar{M}:=M / 2 N$,

- $\bar{M}$ is isotropic with respect to the pairing $\bar{\psi}_{N}$, and

- $M$ generates $N$ over $O_{E}$.

We need to determine the isomorphism classes of elements $M$ in $X_{r}$. Let $\bar{X}_{r}$ be the set of maximally isotropic $\mathbb{F}_{2}$-subspaces $\bar{M}$ of $\bar{N}$ such that $\bar{M}$ generates $\bar{N}$ over $\mathbb{F}_{2} \times \mathbb{F}_{2}$. Since the $R$-module structure of $\bar{N}$ is simply an $\mathbb{F}_{2}$-module, the reduction map $M \mapsto \bar{M}$ gives rise to a bijection $X_{r} \simeq \bar{X}_{r}$.

If two elements $M_{1}$ and $M_{2}$ in $X_{r}$ are isomorphic as skew-Hermitian $R$-modules, then any isomorphism between them lifts to an $R$-linear automorphism of $\left(N, \psi_{N}\right)$. Therefore, the set of isomorphism classes of elements in $X_{r}$ is in bijection with the orbit set $\operatorname{Aut}_{R}\left(N, \psi_{N}\right) \backslash X_{r}$. As the $R$-action on $N$ extends uniquely to an 
$O_{E}$-action on $N$, we have $\operatorname{Aut}_{R}\left(N, \psi_{N}\right)=\operatorname{Aut}_{O_{E}}\left(N, \psi_{N}\right)$. The action of the group $\operatorname{Aut}_{O_{E}}\left(N, \psi_{N}\right)$ on $X_{r}$ factors through $\operatorname{Aut}_{\bar{O}_{E}}\left(\bar{N}, \bar{\psi}_{N}\right)$, and the reduction map yields a bijection

$$
\operatorname{Aut}_{O_{E}}\left(N, \psi_{N}\right) \backslash X_{r} \simeq \operatorname{Aut}_{\bar{O}_{E}}\left(\bar{N}, \bar{\psi}_{N}\right) \backslash \bar{X}_{r} .
$$

Proposition 4.2. Let $r$ be a positive integer. There are

$$
\# \operatorname{Aut}_{\bar{O}_{E}}\left(\bar{N}, \bar{\psi}_{N}\right) \backslash \bar{X}_{r} .
$$

non-isomorphic self-dual skew-Hermitian $R$-modules $M$ such that $M \simeq R^{r}$ as $R$-modules.

We now describe the set $\operatorname{Aut}_{\bar{O}_{E}}\left(\bar{N}, \bar{\psi}_{N}\right) \backslash \bar{X}_{r}$. Let $\bar{N}=\bar{N}^{1} \oplus \bar{N}^{2}$ be the decomposition induced by $\bar{O}_{E}=\mathbb{F}_{2} \times \mathbb{F}_{2}$, and let $p_{i}: \bar{N} \rightarrow \bar{N}^{i}$ be the $i$ th projection for $i=1,2$. Fix a basis $e_{1}, \ldots, e_{r}$ for $\bar{N}^{1}$ and a basis $f_{1}, \ldots, f_{r}$ for $\bar{N}^{2}$ such that $\bar{\psi}_{N}\left(e_{i}, f_{j}\right)=\delta_{i, j}$ for all $i, j=1, \ldots, r$. Using the basis $\left\{e_{i}, f_{i}\right\}_{i=1, \ldots, r}$ we identify the $\mathbb{F}_{2}$-vector space $\bar{N}$ with the $\mathbb{F}_{2}$-space $\mathbb{F}_{2}^{2 r}$ of column vectors. Let $\bar{M}$ be an $\mathbb{F}_{2}$-vector space of $\bar{N}$ of dimension $r$. Choose a basis $v_{1}, \ldots, v_{r}$ for $\bar{M}$. The subspace $\bar{M}$ generates $\bar{N}$ over $\mathbb{F}_{2} \times \mathbb{F}_{2}$ if and only if $p_{i}(\bar{M})=\bar{N}^{i}$ for $i=1,2$. In this case, after a unique change of basis we may assume that $v_{i}=e_{i}+u_{i}$ for $i=1, \ldots, r$ and $u_{1}, \ldots, u_{r}$ forms a basis for $\bar{N}^{2}$. Write $u_{j}=\sum_{i} u_{i j} f_{i}$ and let $U:=\left(u_{i j}\right) \in \mathrm{GL}_{r}\left(\mathbb{F}_{2}\right)$. The matrix $U$ is uniquely determined by $\bar{M}$. One computes

$$
\bar{\psi}_{N}\left(e_{i}+u_{i}, e_{j}+u_{j}\right)=\bar{\psi}_{N}\left(e_{i}, u_{j}\right)-\bar{\psi}_{N}\left(e_{j}, u_{i}\right)=u_{i j}-u_{j i} .
$$

It follows that $\bar{M}$ is isotropic for $\bar{\psi}_{N}$ if and only if the matrix $U$ is symmetric. This shows $\bar{X}_{r} \simeq S_{r}$. Recall that $S_{r}$ denotes in the set of all symmetric matrices in $\mathrm{GL}_{r}\left(\mathbb{F}_{2}\right)$ (Section 1). With respect to the basis $\left\{e_{i}, f_{i}\right\}$ the automorphism group $\operatorname{Aut}_{\bar{O}_{E}}\left(\bar{N}, \bar{\psi}_{N}\right)$ of is

$$
\left\{\left(\begin{array}{cc}
A^{-1} & 0 \\
0 & A^{t}
\end{array}\right) ; A \in \mathrm{GL}_{r}\left(\mathbb{F}_{2}\right)\right\} \simeq \mathrm{GL}_{r}\left(\mathbb{F}_{2}\right), \quad\left(\begin{array}{cc}
A^{-1} & 0 \\
0 & A^{t}
\end{array}\right) \mapsto A^{-1} .
$$

From the formula

$$
\left(\begin{array}{cc}
A^{-1} & 0 \\
0 & A^{t}
\end{array}\right)\left(\begin{array}{c}
I_{r} \\
U
\end{array}\right)=\left(\begin{array}{c}
I_{r} \\
A^{t} U A
\end{array}\right) A^{-1}
$$


the action of the group $\mathrm{GL}_{r}\left(\mathbb{F}_{2}\right) \simeq \operatorname{Aut}_{\bar{O}_{E}}\left(\bar{N}, \bar{\psi}_{N}\right)$ on $S_{r}$, transported from the action on $\bar{X}_{r}$, is given by

$$
A \cdot U=A^{-t} U A^{-1}, \quad \forall A \in \mathrm{GL}_{r}\left(\mathbb{F}_{2}\right), U \in S_{r} .
$$

We have shown the following proposition.

Proposition 4.3. Notation as above. There is a bijection

$$
\operatorname{Aut}_{\bar{O}_{E}}\left(\bar{N}, \bar{\psi}_{N}\right) \backslash \bar{X}_{r} \simeq \mathrm{GL}_{r}\left(\mathbb{F}_{2}\right) \backslash S_{r} .
$$

Note that $S_{r} / \sim$, the set of equivalence classes (see Section 1), is the orbit set $\mathrm{GL}_{r}\left(\mathbb{F}_{2}\right) \backslash S_{r}$.

Proposition 4.4 (Witt Cancellation). Let $M_{1}$ and $M_{2}$ be two self-dual skewHermitian R-modules. Suppose there is an isomorphism

$$
M_{1} \oplus L_{h}^{\oplus s} \simeq M_{2} \oplus L_{h}^{\oplus s}
$$

of skew-Hermitian $R$-modules for some integer $s \geq 0$. Then $M_{1}$ is isometric to $\mathrm{M}_{2}$.

Proof. By Lemma 4.1 (2), we have decompositions $M_{1}=M_{1}^{\prime} \oplus L_{h}^{\oplus s^{\prime}}$ and $M_{2}=M_{2}^{\prime} \oplus L_{h}^{\oplus s^{\prime \prime}}$ as skew-Hermitian modules such that $M_{1}^{\prime}$ and $M_{2}^{\prime}$ are free as $R$ modules. Note that $s^{\prime \prime}=s^{\prime}$. Replacing $M_{1}$ and $M_{2}$ by $M_{1}^{\prime}$ and $M_{2}^{\prime}$, respectively, we may assume that $M_{1} \simeq M_{2} \simeq R^{r}$ as $R$-modules. Write $\psi_{i}$ for the pairings on $M_{i} \oplus L_{h}^{\oplus s}$, for $i=1,2$. Let $N_{i}$ be the smallest $O_{E}$-module in the vector space $E \otimes_{R} M_{i}$ containing $M_{i}$. Then $N_{i} \oplus L_{h}^{\oplus s}$ is the smallest $O_{E}$-module in the vector space $E \otimes_{R}\left(M_{i} \oplus L_{h}^{\oplus s}\right)$ containing $M_{i} \oplus L_{h}^{\oplus s}$. Put $\psi_{i}^{\prime}:=2 \psi_{i}$, which is a $\mathbb{Z}_{2}$-valued skew-Hermitian form on $N_{i} \oplus L_{h}^{\oplus s}$. The pairing $\psi_{i}^{\prime}$ induces a non-degenerate pairing $\overline{\psi_{i}^{\prime}}$ on the $\mathbb{F}_{2}$-vector space

$$
\left(N_{i} \oplus L_{h}^{\oplus s}\right) /\left(2 N_{i} \oplus L_{h}^{\oplus s}\right) \simeq N_{i} / 2 N_{i}=: \bar{N}_{i},
$$

and the subspace

$$
\left(M_{i} \oplus L_{h}^{\oplus s}\right) /\left(2 N_{i} \oplus L_{h}^{\oplus s}\right) \simeq M_{i} / 2 N_{i}=: \bar{M}_{i}
$$

is a maximal isotropic subspace with respect to $\overline{\psi_{i}^{\prime}}$.

Let $\alpha: M_{1} \oplus L_{h}^{\oplus s} \simeq M_{2} \oplus L_{h}^{\oplus s}$ be an isomorphism of skew-Hermitian $R$-modules. The map $\alpha$ lifts to an isomorphism $\beta: N_{1} \oplus L_{h}^{\oplus s} \simeq N_{2} \oplus L_{h}^{\oplus s}$, and $\beta$ induces an isomorphism $\bar{\beta}: \bar{N}_{1} \simeq \bar{N}_{2}$ of symplectic $\mathbb{F}_{2}$-spaces such that $\bar{\beta}\left(\bar{M}_{1}\right)=\bar{M}_{2}$. 
We lift Lagrangian bases $\left\{x_{i}\right\}$ for $\left.\bar{N}_{1}\right)$ and $\left\{\bar{\beta}\left(x_{i}\right)\right\}$ for $\bar{N}_{2}$ to Lagrangian bases $X_{i}$ for $N_{1}$ and $Y_{i}$ for $N_{2}$, respectively. The map $\gamma: N_{1} \simeq N_{2}$ which sends $X_{i}$ to $Y_{i}$ is an isomorphism of skew-Hermitian $R$-modules. Since $\gamma\left(2 N_{1}\right)=2 N_{2}$ and $\gamma\left(\bar{M}_{1}\right)=\bar{M}_{2}$, it gives an isomorphism from $M_{1}$ to $M_{2}$. This proves the proposition.

Remark 4.5. Proposition 4.4 (also Proposition 5.7) is not covered by a general Witt type cancellation theorem [1, Theorem 3] proved by Bayer-Fluckiger and Fainsilber, as the condition $a+\bar{a}=1$ for some $a \in R$ is not fulfilled.

Corollary 4.6. Let $M$ be a self-dual skew-Hermitian $R$-module of $\mathbb{Z}_{2}$-rank $2 n$. Then there are unique non-negative integers $r$ and $s$ with $r+s=n$ and a self-duel skew-Hermitian $R$-module $M_{1}$ which is free of rank $r$ such that

$$
M \simeq M_{1} \oplus L_{h}^{\oplus s}
$$

Moreover, $M_{1}$ is uniquely determined by $M$ up to isomorphism.

Proof. This follows immediately from Lemma 4.1 (2) and Proposition 4.4.

By Corollary 4.6 and Propositions 4.2 and 4.3, Theorem 1.1 is proved.

4.5. It is well-known that the set $S_{r} / \sim$ parametrizes equivalence classes of nondegenerate symmetric $\mathbb{F}_{2}$-spaces $(W, \varphi)$ of dimension $r$. We use the notation (1) to indicate the one-dimensional non-degenerate symmetric space $\mathbb{F}_{2}$ with the pairing $\varphi\left(e_{1}, e_{1}\right)=1$, and write

$$
\left(\begin{array}{ll}
0 & 1 \\
1 & 0
\end{array}\right)
$$

for the two-dimensional non-degenerate symmetric space $\mathbb{F}_{2} \oplus \mathbb{F}_{2}$ with the pairing

$$
\varphi\left(e_{1}, e_{1}\right)=\varphi\left(e_{2}, e_{2}\right)=0, \quad \varphi\left(e_{1}, e_{2}\right)=1 .
$$

Lemma 4.7. Let $(W, \varphi)$ be a symmetric space over $\mathbb{F}_{2}$ of dimension $r \geq 1$. If $r$ is odd, then

$$
(W, \varphi) \simeq(1) \oplus\left(\begin{array}{ll}
0 & 1 \\
1 & 0
\end{array}\right) \oplus \cdots \oplus\left(\begin{array}{ll}
0 & 1 \\
1 & 0
\end{array}\right)
$$


If $r$ is even, then either

$$
\begin{aligned}
& (W, \varphi) \simeq\left(\begin{array}{ll}
0 & 1 \\
1 & 0
\end{array}\right) \oplus \cdots \oplus\left(\begin{array}{ll}
0 & 1 \\
1 & 0
\end{array}\right), \text { or } \\
& (W, \varphi) \simeq\left(\begin{array}{ll}
0 & 1 \\
1 & 0
\end{array}\right) \oplus \cdots \oplus\left(\begin{array}{ll}
0 & 1 \\
1 & 0
\end{array}\right) \oplus(1) \oplus(1) .
\end{aligned}
$$

In particular, we have

$$
\# S_{r} / \sim= \begin{cases}1, & \text { if } r \text { is odd } \\ 2, & \text { if } r \text { is even }\end{cases}
$$

Proof. Denote by $N(W)$ the set of vectors $x \in W$ such that $\varphi(x, x)=0$. It is easy to show that $N(W)$ is a subspace. Let $N(W)_{n}$ be the null subspace of $N(W)$ and $W_{2} \subset N(W)$ be a complement of $N(W)_{n}$. Then $W_{2}$ is a maximal non-degenerate subspace in $N(W)$ and it is easy to show

$$
W_{2} \simeq\left(\begin{array}{ll}
0 & 1 \\
1 & 0
\end{array}\right) \oplus \cdots \oplus\left(\begin{array}{ll}
0 & 1 \\
1 & 0
\end{array}\right) .
$$

Let $W_{1}:=W_{2}^{\perp} \subset W$ be the orthogonal complement of $W_{2}$; one has $W=W_{1} \oplus W_{2}$ and $N(W)=N\left(W_{1}\right) \oplus W_{2}$. It follows that $N(W)_{n}=N\left(W_{1}\right)=W\left(W_{1}\right)_{n}$ in $W_{1}$. We have $\operatorname{dim} W_{1} \leq \operatorname{dim} N\left(W_{1}\right)+1$, as if one has $y \neq x$ with $\varphi(x, x)=1$ and $\varphi(y, y)=1$ then $\varphi(x+y, x+y)=0$. It follows that $\operatorname{dim} N\left(W_{1}\right) \leq 1$, otherwise $W_{1}$ would be degenerate. Therefore, $\operatorname{dim} W_{1} \leq 2$. As there are no vectors $x, y \in W_{1}$ such that $\varphi(x, x)=\varphi(y, y)=0$ and $\varphi(x, y)=1$, the symmetric space $\left(W_{1}, \varphi\right)$ is isomorphic to the direct sum of copies of (1). Therefore, the lemma follows.

\section{Proof of Theorem 1.2 .}

5.1. In this section we assume that $p \equiv 3(\bmod 8)$. Thus, the algebra $E$ is an unramified quadratic field extension of $\mathbb{Q}_{2}$. Recall that

$$
R=\mathbb{Z}_{2}[\omega]=\mathbb{Z}_{2}[X] /\left(X^{2}+2 X+p+1\right) \subset O_{E}=\mathbb{Z}_{2}[\alpha] /\left(X^{2}+X+(p+1) / 4\right),
$$

$\omega=\pi-1, \alpha=\omega / 2$ and $\pi \in R$ is an element with $\pi^{2}=-p$. Let $(M, \psi)$ be a skew-Hermitian $R$-module of $\mathbb{Z}_{2}$-rank $2 n$, and let $V:=M \otimes_{\mathbb{Z}_{2}} \mathbb{Q}_{2}$. There is a unique non-degenerate $E$-valued skew-Hermitian form

$$
\langle,\rangle: V \times V \rightarrow E
$$


such that $\langle a x, b y\rangle=a \bar{b}\langle x, y\rangle$ for all $a, b \in E$ and $x, y \in V$, and $\psi(x, y)=$ $\operatorname{Tr}_{E / \mathbb{Q}_{2}}\langle x, y\rangle$ for all $x, y \in V$. Since $E / \mathbb{Q}_{2}$ is unramified, the inverse different $\mathcal{D}_{E / \mathbb{Q}_{2}}^{-1}$ is equal to $O_{E}$. Put $():,=\pi\langle$,$\rangle , which is a Hermitian form on V$. Note that $\langle M, M\rangle \subset O_{E}$ if and only if $(M, M) \subset O_{E}$, as the element $\pi=1+\omega$ lands in $1+2 O_{E}=R^{\times} \subset O_{E}^{\times}$.

\section{Lemma 5.1.}

(1) One has $(M, M) \subset 2^{-1} R$. Under the assumption that $M$ is self-dual with respect to the pairing $\psi$, the condition $(M, M) \subset O_{E}$ holds if and only if $M$ is invariant under the $O_{E}$-action.

(2) The $R$-lattice $M$ is self-dual with respect to the pairing $\psi$ if and only if $M$ is self-dual with respect to the pairing $2($, ).

Proof. (1) The element $\langle x, y\rangle$ for $x, y \in M$ satisfies the property $\operatorname{Tr}_{E / \mathbb{Q}_{2}}(\langle x, y\rangle R) \subset$ $\mathbb{Z}_{2}$. Therefore, it lands in the dual lattice $R^{\vee}$ of $R$ for the pairing $(a, b) \mapsto$ $\operatorname{Tr}_{E / \mathbb{Q}_{2}}(a b)$. It is easy to check that $R^{\vee}=2^{-1} R$, and hence the first part is proved.

Let $\widetilde{M}$ be the $O_{E}$-submodule in $V$ generated by $M$. If $M$ is invariant under the $O_{E}$-action, then $\langle M, M\rangle \subset O_{E}^{\vee}=O_{E}$. Conversely, if $\langle M, M\rangle \subset O_{E}$, then $\langle\widetilde{M}, \widetilde{M}\rangle \subset O_{E}$. This yields $\psi(\widetilde{M}, \widetilde{M}) \subset \mathbb{Z}_{2}$. As $M$ is self-dual, one has $\widetilde{M}=M$. This proves (1).

(2) For any element $x \in V$, one has

$$
(x, M) \in 2^{-1} R \Longleftrightarrow \psi(x, M) \in \mathbb{Z}_{2} .
$$

Therefore, the assertion follows.

From now on, we assume that $M$ is self-dual with respect to the pairing $\psi$.

Lemma 5.2. One has $N_{E / \mathbb{Q}_{2}}\left(R^{\times}\right)=\mathbb{Z}_{2}^{\times}$.

Proof. We use the following basic fact in number theory; see [10, Corollary, p. 7]. Suppose $E / F$ is a unramified finite extension of non-Archimedean local fields. For any integer $i \geq 1$, let $U_{E}^{(i)}:=1+\pi_{E}^{i} O_{E} \subset O_{E}^{\times}$and $U_{F}^{(i)}:=1+\pi_{F}^{i} O_{F} \subset$ $O_{F}^{\times}$be the $i$ th principal congruence subgroups of $O_{E}^{\times}$and $O_{F}^{\times}$, respectively. Then we have $N_{E / F}\left(U_{E}^{(i)}\right)=U_{F}^{(i)}$.

Since $R^{\times}=1+2 O_{E}$ and $\mathbb{Z}_{2}^{\times}=1+2 \mathbb{Z}_{2}$, the lemma follows. 
5.2. We define three self-dual skew-Hermitian $R$-modules $L_{0}, L_{1}, H$ as follows.

(i) The $R$-module $L_{0}$ is $O_{E}$ and the pairing $\psi_{0}$ is defined by $\psi_{0}(1, \omega / 2)=1$. Write $\omega^{\prime}$ for the conjugate of $\omega$. To see this defines a skew-Hermitian $R$-module, one checks that

$$
\psi_{0}\left(\omega^{\prime} / 2,1\right)=\psi_{0}((-2-\omega) / 2,1)=\psi_{0}(-\omega / 2,1)=\psi_{0}(1, \omega / 2) .
$$

(ii) The $R$-module $L_{1}$ is $R$ and the pairing $\psi_{1}$ is defined by $\psi_{1}(1, \omega)=1$. Similarly, one checks that $\psi_{1}(1, \omega)=\psi_{1}\left(\omega^{\prime}, 1\right)$ as $(5.2)$.

(iii) The $R$-module $H$ is $R \oplus R$ with standard basis $e_{1}=(1,0)$ and $e_{2}=(0,1)$.

The pairing $\psi_{H}$ is defined by

$$
\begin{gathered}
\psi_{H}\left(e_{1}, \omega e_{1}\right)=\psi_{H}\left(e_{1}, e_{2}\right)=\psi_{H}\left(e_{2}, \omega e_{2}\right)=\psi_{H}\left(\omega e_{1}, \omega e_{2}\right)=0, \\
\psi_{H}\left(e_{1}, \omega e_{2}\right)=\psi_{H}\left(e_{2}, \omega e_{1}\right)=1 .
\end{gathered}
$$

Note that using the relation $\psi_{H}(\omega x, y)=\psi_{H}\left(x, \omega^{\prime} y\right)$ the pairing $\psi_{H}$ is uniquely determined by any values of

$$
\psi_{H}\left(e_{1}, \omega e_{1}\right), \psi_{H}\left(e_{1}, e_{2}\right), \psi_{H}\left(e_{1}, \omega e_{2}\right) \text {, and } \psi_{H}\left(e_{2}, \omega e_{2}\right) .
$$

One checks that

$$
\psi_{H}\left(\omega^{\prime} e_{1}, e_{2}\right)=\psi_{H}\left((-2-\omega) e_{1}, e_{2}\right)=\psi_{H}\left(e_{2}, \omega e_{1}\right)=1=\psi_{H}\left(e_{1}, \omega e_{2}\right) .
$$

So (5.3) defines a skew-Hermitian $R$-module.

By Theorem 3.1, there are unique non-negative integers $r$ and $s$ with $r+s=n$ such that

$$
M \simeq R^{r} \oplus O_{E}^{s}
$$

as $R$-modules. If one has a decomposition

$$
M \simeq L_{1}^{\oplus r_{1}} \oplus H^{\oplus r_{2}} \oplus L_{0}^{\oplus s^{\prime}}
$$

as skew-Hermitian $R$-modules for some non-negative integers $r_{1}, r_{2}$ and $s^{\prime}$, then $r_{1}+2 r_{2}=r$ and $s^{\prime}=s$.

5.3. Let $M_{0} \subset M$ be the $R$-submodule defined by

$$
M_{0}:=\{x \in M \mid \omega x \in 2 M\} .
$$

Write $\bar{M}:=M / 2 M$ and $\bar{M}_{0}:=M_{0} / 2 M$. Let

$$
\bar{\psi}: \bar{M} \times \bar{M} \rightarrow \mathbb{F}_{2}
$$


be the induced non-degenerate alternating pairing. We have a filtration

$$
\omega \bar{M} \subset \bar{M}_{0} \subset \bar{M},
$$

and have the following properties

- $\operatorname{dim}_{\mathbb{F}_{2}} \omega \bar{M}=\operatorname{dim}_{\mathbb{F}_{2}} \bar{M} / \bar{M}_{0}=r$ and $\operatorname{dim}_{\mathbb{F}_{2}} \bar{M}_{0}=2 s+r$, and

- $\omega \bar{M}$ is an isotropic subspace with respect to the pairing $\bar{\psi}$.

For any element $x$ in $M$, write $\bar{x}$ for the image of $x$ in $\bar{M}$.

Lemma 5.3. Let $M$ be a self-dual skew-Hermitian R-module.

(1) If there exists an element $x \in M$ such that $\bar{\psi}(\bar{x}, \omega \bar{x}) \neq 0$, then the $R$ submodule $M_{1}$ generated by $x$, with the pairing $\left.\psi\right|_{M_{1}}$ restricted on $M_{1}$, is isomorphic to $\left(L_{1}, \psi_{1}\right)$ as skew-Hermitian $R$-modules. Moreover, we have the decomposition

$$
M=M_{1} \oplus M_{1}^{\perp}
$$

as skew-Hermitian $R$-modules.

(2) If there exists an element $x \in M_{0} \subset M$ such that $\bar{\psi}(\bar{x}, \overline{\omega / 2 x}) \neq 0$, then the $R$-submodule $M_{1}$ generated by $x$ and $\omega / 2 x$, with the pairing $\left.\psi\right|_{M_{1}}$ restricted on $M_{1}$, is isomorphic to $\left(L_{0}, \psi_{0}\right)$ as skew-Hermitian $R$-modules. Moreover, we have the decomposition

$$
M=M_{1} \oplus M_{1}^{\perp}
$$

as skew-Hermitian R-modules.

Proof. (1) We have $\psi(x, \omega x)=a \in \mathbb{Z}_{2}^{\times}$. By Lemma 5.2, there is an element $\lambda \in R^{\times}$such that $N(\lambda)=a^{-1}$. Replacing $x$ by $\lambda x$, we get $\psi(x, \omega x)=1$. Since $M_{1}$ is self-dual and $R$-invariant, the orthogonal complement $M_{1}^{\perp}$ is $R$-invariant and we have $M=M_{1} \oplus M_{1}^{\perp}$. This proves (1).

(2) Using the same argument as (1), we can choose an element $x \in M_{1}$ such that $\psi(x, \omega / 2 x)=1$ and we have the decomposition $M=M_{1} \oplus M_{1}^{\perp}$. It is clear that we have an isometry $\left(M_{1},\left.\psi\right|_{M_{1}}\right) \simeq\left(L_{0}, \psi_{0}\right)$. This proves $(2)$.

Lemma 5.4. There is a decomposition as skew-Hermitian R-modules

$$
M \simeq M_{1} \oplus L_{0}^{\oplus s}
$$

where $M_{1}$ is a free $R$-submodule of rank $r$ which is self-dual with respect to the pairing $\psi$. 
Proof. Let $M_{0} \subset M$ be the submodule defined as (5.4). It is clear that $\bar{\psi}\left(\omega \bar{M}, \bar{M}_{0}\right)=0$. It follows from the non-degeneracy of $\bar{\psi}$ that there are vectors $x_{1}, \ldots, x_{r} \in \omega \bar{M}$ and $y_{1}, \ldots, y_{r} \in \bar{M}$ such that

$$
\bar{\psi}\left(x_{i}, x_{j}\right)=\bar{\psi}\left(y_{i}, y_{j}\right)=0, \text { and } \bar{\psi}\left(x_{i}, y_{j}\right)=\delta_{i, j}
$$

for all $i, j=1, \ldots, r$. Put $W_{1}:=<y_{1}, \ldots, y_{r}>_{\mathbb{F}_{2}}$ and $W_{2}:=\left(\omega \bar{M} \oplus W_{1}\right)^{\perp}$. We have $\bar{M}=\omega \bar{M} \oplus W_{1} \oplus W_{2}$. It follows from

$$
\bar{\psi}\left(\omega W_{2}, \bar{M}\right)=\bar{\psi}\left(W_{2},(-2-\omega) \bar{M}\right)=0
$$

that $\omega W_{2}=0$, and hence $W_{2} \subset \bar{M}_{0}$. It follows from the dimension counting that $\bar{M}_{0}=\omega \bar{M} \oplus W_{2}$. This shows that the pairing $\bar{\psi}$ is non-degenerate on $\bar{M}_{0} / \omega \bar{M}$. We can choose elements $z_{1}, \ldots, z_{s}$ in $M_{0}$ such that the $O_{E}$-submodule $M_{2}=<z_{1}, \ldots, z_{s}>_{O_{E}} \subset M_{0}$ is mapped onto $W_{2}$. The module $M_{2}$ is self-dual with respect to $\psi$. By Lemma 5.1, $M_{2}$ is a unimodular Hermitian module over $O_{E}$ for the pairing (, ), and hence $M_{2}$ is a direct sum of $O_{E}$-rank one Hermitian submodule, as $E / \mathbb{Q}_{2}$ is unramified. Therefore, $M_{2} \simeq L_{0}^{\oplus s}$ as skew-Hermitian $R$ modules. Put $M_{1}:=M_{2}^{\perp}$. We have $M=M_{1} \oplus M_{2}$ as skew-Hermitian modules, and $M_{1}$ is a free $R$-submodule of rank $r$ which is self-dual with respect to the pairing $\psi$.

5.4. Now we classify self-dual skew-Hermitian $R$-modules $(M, \psi)$ in the case where $M \simeq R^{r}$, where $r$ is a positive integer. Let $N$ be the smallest $O_{E}$-module in $V$ containing $M$, and let $N^{\prime}$ be the largest $O_{E^{-}}$submodule in $M$. We have $N^{\prime}=2 N \supset 2 M$. Since $N^{\prime} / 2 M$ is a maximal isotropic $\mathbb{F}_{2}$-subspace of dimension $r$ with respect to $\bar{\psi}$, the polarization type of $\psi$ on $N^{\prime}$ is $(2, \ldots, 2)$. Put $\psi_{N}:=$ $2 \psi$. We have $\psi_{N}(N, N) \subset \mathbb{Z}_{2}$, and $N$ is self-dual with respect to the pairing $\psi_{N}$. Note that $\left(N, \psi_{N}\right) \simeq\left(L_{0}, \psi_{0}\right)^{\oplus r}$ by Lemma 5.4. Put $\bar{N}=N / 2 N$ and let $\bar{\psi}_{N}: \bar{N} \times \bar{N} \rightarrow \mathbb{F}_{2}$ be the induced non-degenerate pairing. We have

$$
2 N \subset M \subset N, \quad \operatorname{dim}_{\mathbb{F}_{2}} \bar{M}=r
$$

and $\bar{M}$ is isotropic for $\bar{\psi}_{N}$, where $\bar{M}:=M / 2 N$. Note that $M$ generates $N$ over $O_{E}$, or equivalently, the subspace $\bar{M}$ generates $\bar{N}$ over $\bar{O}_{E}:=O_{E} / 2 O_{E}=\mathbb{F}_{4}$.

Suppose $M_{1}$ is another self-dual skew-Hermitian $R$-module such that $M_{1} \simeq R^{r}$. Define $N_{1}$ and $\psi_{N_{1}}$ similarly. As $\left(N_{1}, \psi_{N_{1}}\right) \simeq\left(N, \psi_{N}\right)$, we choose an isomorphism $\alpha:\left(N_{1}, \psi_{N_{1}}\right) \simeq\left(N, \psi_{N}\right)$ of skew-Hermitian $R$-modules. The image $M^{\prime}=\alpha\left(M_{1}\right)$ is an $R$-module which satisfies the same property as $M$ does (5.6). 
Now we fix the self-dual skew-Hermitian $R$-module $\left(N, \psi_{N}\right)$. Similarly to $\S 4.4$, let $X_{r}$ be the set of all $R$-submodules $M$ of $N$ such that

- $2 N \subset M \subset N$ and $\operatorname{dim}_{\mathbb{F}_{2}} \bar{M}=r$,

- $\bar{M}$ is isotropic with respect to the pairing $\bar{\psi}_{N}$, and

- $M$ generates $N$ over $O_{E}$.

We need to determine the isomorphism classes of elements $M$ in $X_{r}$. Let $\bar{X}_{r}$ be the set of maximally isotropic $\mathbb{F}_{2}$-subspaces $\bar{M}$ of $\bar{N}$ such that $\bar{M}$ generates $\bar{N}$ over $\mathbb{F}_{4}$. Since the $R$-module structure of $\bar{N}$ is simply an $\mathbb{F}_{2}$-module, the reduction map $M \mapsto \bar{M}$ gives rise to a bijection $X_{r} \simeq \bar{X}_{r}$.

Using the same argument as in $\S 4.4$, the set of isomorphism classes of elements in $X_{r}$ is in bijection with the orbit set $\operatorname{Aut}_{O_{E}}\left(N, \psi_{N}\right) \backslash X_{r}$, and the reduction map induces the bijection

$$
\operatorname{Aut}_{O_{E}}\left(N, \psi_{N}\right) \backslash X_{r} \simeq \operatorname{Aut}_{\mathbb{F}_{4}}\left(\bar{N}, \bar{\psi}_{N}\right) \backslash \bar{X}_{r}
$$

Proposition 5.5. Let $r$ be a positive integer. There are

$$
\# \operatorname{Aut}_{\mathbb{F}_{4}}\left(\bar{N}, \bar{\psi}_{N}\right) \backslash \bar{X}_{r}
$$

non-isomorphic self-dual skew-Hermitian $R$-modules $M$ such that $M \simeq R^{r}$ as $R$-modules.

We now describe the orbit set $\operatorname{Aut}_{\bar{O}_{E}}\left(\bar{N}, \bar{\psi}_{N}\right) \backslash \bar{X}_{r}$. Let $V_{0}=\mathbb{F}_{4}^{r}$, viewed as the space of column vectors, together with the standard non-degenerate Hermitian form $(,)_{0}$ defined by

$$
\left(\left(x_{i}\right),\left(y_{i}\right)\right)_{0}=\sum_{i=1}^{r} x_{i} \bar{y}_{i},
$$

where $y \mapsto \bar{y}$ is the non-trivial automorphism on $\mathbb{F}_{4}$. Choose an element $\epsilon \in \mathbb{F}_{4}^{\times}$ such that $\epsilon+\bar{\epsilon}=0$ (in fact $\epsilon=1$ ) and put $\langle x, y\rangle_{0}:=\operatorname{Tr}_{\mathbb{F}_{4} / \mathbb{F}_{2}} \epsilon(x, y)_{0}$, for $x, y \in V_{0}$. Then we have an isomorphism $\left(V_{0},\langle,\rangle_{0}\right) \simeq\left(\bar{N}, \bar{\psi}_{N}\right)$ as skew-Hermitian modules over $\mathbb{F}_{4}$ and we may identify $\left(\bar{N}, \bar{\psi}_{N}\right)$ with $\left(V_{0},\langle,\rangle_{0}\right)$. Let $U(r)$ be the unitary group over $\mathbb{F}_{2}$ associated to the Hermitian space $\left(V_{0},(,)_{0}\right)$; one has

$$
U(r)\left(\mathbb{F}_{2}\right)=\operatorname{Aut}_{\mathbb{F}_{4}}\left(V_{0},(,)_{0}\right)=\operatorname{Aut}_{\mathbb{F}_{4}}\left(V_{0},\langle,\rangle_{0}\right)
$$

Write any matrix $U$ in $\mathrm{GL}_{r}\left(\mathbb{F}_{4}\right)$ as $\left(u_{1}, \ldots, u_{r}\right)$, where $u_{i} \in V_{0}$. The map

$$
U=\left(u_{1}, \ldots, u_{r}\right) \mapsto W=<u_{1}, \ldots, u_{r}>_{\mathbb{F}_{2}}
$$


induces a bijection between $\mathrm{GL}_{r}\left(\mathbb{F}_{4}\right) / \mathrm{GL}_{r}\left(\mathbb{F}_{2}\right)$ and the set $Z_{r}$ of $r$-dimensional $\mathbb{F}_{2}$-subspace $W \subset V_{0}$ which spans $V_{0}$ over $\mathbb{F}_{4}$. Note that $\operatorname{Tr}_{\mathbb{F}_{4} / \mathbb{F}_{2}}(x, y)_{0}=0$ if and only if $(x, y)_{0} \in \mathbb{F}_{2}$. It follows that a subspace $W \in Z_{r}$ is isotropic with respect to $\langle,\rangle_{0}$ if and only if $\bar{U}^{t} U \in \mathrm{GL}_{r}\left(\mathbb{F}_{2}\right)$ for any matrix $U$ mapping to $W$. Let $Y_{r} \subset \mathrm{GL}_{r}\left(\mathbb{F}_{4}\right)$ be the subset consisting of matrices $U$ such that $\bar{U}^{t} U \in \mathrm{GL}_{r}\left(\mathbb{F}_{2}\right)$. The action of the group $U(r)\left(\mathbb{F}_{2}\right)$ on the set $\mathrm{GL}_{r}\left(\mathbb{F}_{4}\right) / \mathrm{GL}_{r}\left(\mathbb{F}_{2}\right)$, transported from the action of the group $\operatorname{Aut}_{\bar{O}_{E}}\left(\bar{N}, \bar{\psi}_{N}\right)=\operatorname{Aut}_{\mathbb{F}_{4}}\left(V_{0},\langle,\rangle_{0}\right)$ on the set $\bar{X}_{r}$, is simply the left translation. Thus, there is a bijection

$$
\operatorname{Aut}_{\bar{O}_{E}}\left(\bar{N}, \bar{\psi}_{N}\right) \backslash \bar{X}_{r} \simeq U(r)\left(\mathbb{F}_{2}\right) \backslash Y_{r} / \mathrm{GL}_{r}\left(\mathbb{F}_{2}\right) .
$$

Lemma 5.6. The map $\pi: Y_{r} \rightarrow \mathrm{GL}_{r}\left(\mathbb{F}_{2}\right)$ defined by $U \mapsto \bar{U}^{t} U$ induces the bijection

$$
U(r)\left(\mathbb{F}_{2}\right) \backslash Y_{r} / \mathrm{GL}_{r}\left(\mathbb{F}_{2}\right) \simeq S_{r} / \sim
$$

Proof. Since the matrix $\bar{U}^{t} U$ is Hermitian, one has $\pi\left(Y_{r}\right) \subset S_{r}$. Each nonempty fiber of $\pi$ is a principal homogeneous space of $U(r)\left(\mathbb{F}_{2}\right)$. Thus, the induced map $\pi: U(r)\left(\mathbb{F}_{2}\right) \backslash Y_{r} \rightarrow S_{r}$ is injective. One easily checks $\pi(U P)=P^{t} \pi(U) P$ for $P \in \mathrm{GL}_{r}\left(\mathbb{F}_{2}\right)$. Thus, we have the injection map

$$
U(r)\left(\mathbb{F}_{2}\right) \backslash Y_{r} / \mathrm{GL}_{r}\left(\mathbb{F}_{2}\right) \rightarrow S_{r} / \sim
$$

It suffices to show that the map $\pi: Y_{r} \rightarrow S_{r} / \sim$ is surjective. By Lemma 4.7, any matrix $A$ in $S_{r}$ is equivalent to a matrix with diagonal boxes either (1) or

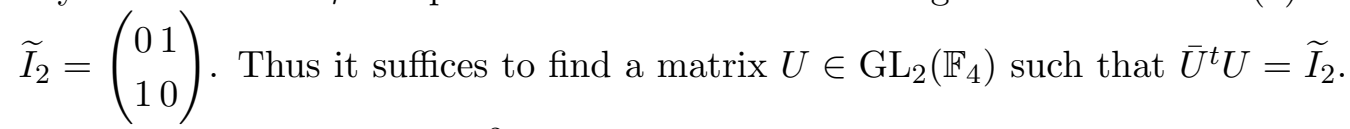
Note that $\mathbb{F}_{4}=\mathbb{F}_{2}[\beta]$ with $\beta^{2}+\beta+1=0$. Take

$$
U=\left(\begin{array}{l}
\beta \beta+1 \\
1 \beta+1
\end{array}\right)
$$

and get $\bar{U}^{t} U=\widetilde{I}_{2}$. This proves the lemma.

Proposition 5.7 (Witt Cancellation). Let $M_{1}$ and $M_{2}$ be two self-dual skewHermitian $R$-modules. Suppose there is an isomorphism

$$
M_{1} \oplus L_{0}^{\oplus s} \simeq M_{2} \oplus L_{0}^{\oplus s}
$$

of skew-Hermitian $R$-modules for some integer $s \geq 0$. Then $M_{1}$ is isometric to $M_{2}$. 
Proof. The proof is the same as that of Proposition 4.4. Note that one can lift the isomorphism $\bar{\beta}:\left(\bar{N}_{1}, \bar{\psi}_{1}\right) \simeq\left(\bar{N}_{2}, \bar{\psi}_{2}\right)$ to an isomorphism $\gamma:\left(N_{1}, \psi_{1}\right) \simeq$ $\left(N_{2}, \psi_{2}\right)$. This is because $\operatorname{Isom}_{R}\left(\left(N_{1}, \psi_{1}\right),\left(N_{2}, \psi_{2}\right)\right)$ is the set of $\mathbb{Z}_{2}$-points of a smooth scheme over $\mathbb{Z}_{2}$ (in fact a trivial torsor of a smooth group scheme over $\left.\mathbb{Z}_{2}\right)$.

Corollary 5.8. Let $M$ be a self-dual skew-Hermitian $R$-module of $\mathbb{Z}_{2}$-rank $2 n$. Then there are unique non-integers $r$ and $s$ with $r+s=n$ and a self-duel skewHermitian R-module $M_{1}$ which is free of rank $r$ such that

$$
M \simeq M_{1} \oplus L_{0}^{\oplus s} .
$$

Moreover, $M_{1}$ is uniquely determined, up to isomorphism, by $M$.

Proof. This follows immediately from Lemma 5.4 and Proposition 5.7.

Theorem 1.2 follows from Corollary 5.8, Proposition 5.5, (5.8), and Lemma 5.6.

\section{REFERENCES}

[1] E. Bayer-Fluckiger and L. Fainsilber, Non-unimodular Hermitian forms. Invent. Math. 123 (1996), no. 2, 233-240.

[2] C. W. Curtis and I. Reiner, Methods of representation theory. Vol. I. With applications to finite groups and orders. Pure and Applied Mathematics. A Wiley-Interscience Publication. John Wiley \& Sons, Inc., New York, 1981, 819 pp.

[3] L. Fainsilber and J. Morales, An injectivity result for Hermitian forms over local orders. Illinois J. Math. 43 (1999), no. 2, 391-402.

[4] T. Ibukiyama and T. Katsura, On the field of definition of superspecial polarized abelian varieties and type numbers. Compositio Math. 91 (1994), 37-46.

[5] T. Ibukiyama, T. Katsura and F. Oort, Supersingular curves of genus two and class numbers. Compositio Math. 57 (1986), 127-152.

[6] R. Jacobowitz, Hermitian forms over local fields. Amer. J. Math. 84 (1962), 441-465.

[7] M.-A. Knus, A. Merkurjev, M. Rost, and J.-P. Tignol, The book of involutions. American Mathematical Society, Colloquium Publications, 44. Amer. Math. Soc., 1998, 593 pp.

[8] H. Matsumura, Commutative algebra. Second edition. Mathematics Lecture Note Series, 56. Benjamin/Cummings Publishing, 1980, 313 pp.

[9] D. Mumford, Abelian Varieties. Oxford University Press, 1974.

[10] V. Platonov and A. Rapinchuk, Algebraic groups and number theory. Pure and Applied Mathematics, 139. Academic Press, Inc., Boston, MA, 1994.

[11] I. Reiner, Maximal orders. London Mathematical Society Monographs, No. 5. Academic Press, London-New York, 1975, 395 pp. 
[12] C. Riehm, Hermitian forms over local hereditary orders. Amer. J. Math. 106 (1984), no. 4, 781-800.

[13] W. Scharlau, Quadratic and Hermitian forms. Grundlehren der Mathematischen Wissenschaften 270. Springer-Verlag, Berlin, 1985.

[14] G. Shimura, Arithmetic of Hermitian forms. Doc. Math. 13 (2008), 739-774.

[15] J. Tate, Classes d'isogenie de variétés abéliennes sur un corps fini (d'après T. Honda). Sém. Bourbaki Exp. 352 (1968/69). Lecture Notes in Math., vol. 179, Springer-Verlag, 1971.

[16] J. Tate, Endomorphisms of abelian varieties over finite fields. Invent. Math. 2 (1966), 134144 .

[17] W. C. Waterhouse, Abelian varieties over finite fields. Ann. Sci. École Norm. Sup. (4) 2 (1969), 521-560.

[18] C.-F. Yu, Superspecial abelian varieties over finite prime fields. J. Pure Appl. Algebra 216 (2012), 1418-1427.

\section{Chia-Fu $\mathrm{Yu}$}

Institute of Mathematics, Academia Sinica and NCTS (Taipei Office)

6th Floor, Astronomy Mathematics Building

No. 1, Roosevelt Rd. Sec. 4

Taipei, Taiwan, 10617

E-mail: chiafu@math.sinica.edu.tw 